\title{
Magic moments
}

\section{A. P. Ramirez}

hysicists who study condensed matter are interested in the properties that emerge when many molecules or atoms interact. One such emergent property is crystalline order among atoms; another is its opposite, the glass state, which involves rapid cooling through a melting temperature.

'Frustration', the inability of a system to find a unique ground state, is a common feature of disordered solids. Frustration can also arise in an ordered solid solely from geometric considerations, and this is increasingly being recognized as an organizing principle that governs a wide range of physical phenomena in the collective behaviour of atoms. Yet contrary to the implication of their name, frustrated systems actually have more configurational options than conventional collective systems. The basic concepts of frustrated systems are turning out to have practical uses in areas from microelectronics to drug delivery.

The full reality of a solid is determined not only by the positions of its atoms or molecules, but by their intrinsic local properties - their magnetic, electric or rotational degrees of freedom, or moments. A ferromagnetic crystal, for instance, has two types of order: that of its atoms, and that defined by the mutual alignment of the atomic magnetic moments. When the local-moment order has a lower energy than the crystalline order, the crystalline lattice imposes its symmetry on the local moments - for instance, the magnetic field inside the ferromagnet has the same periodicity as the crystallattice. Alternatively, the local-moment order can possess its own symmetry, dictated by the interactions between moments. For the ferromagnet, this symmetry is simply the mutual alignment of magnetic moments, which is compatible with any crystal symmetry.

What happens when the interaction between moments is not compatible with the crystal symmetry? Take antiferromagnetism, which occurs when the magnetic moments

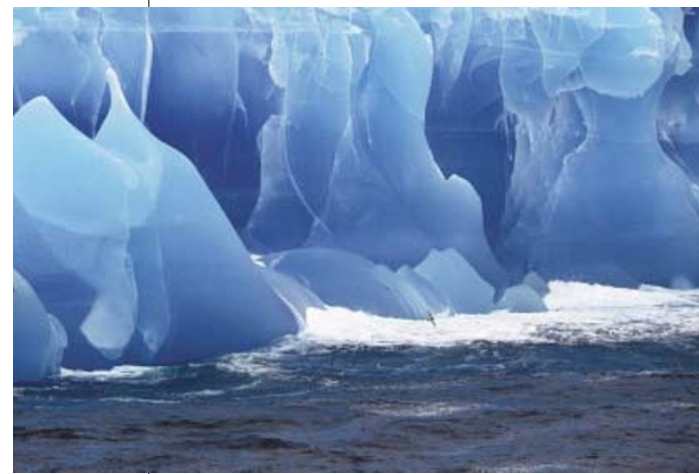

Ice provides the commonest case of frustration. of neighbouring atoms prefer anti-alignment Although such an interaction is compatible with a square lattice, when such moments reside on a triangular lattice there is no simple ordered pattern that satisfies all of the bonds. In this situation, the unsatisfied bond is said to be frustrated. In a system composed of many such triangles, the threefold multiplicity of lowest and equal energy states translates into a large amount of low-energy entropygeometric frustration.

Qualitatively new types of property can emerge when such systems are cooled to temperatures well below the energy of moment-moment interactions. An example is the 'spin-liquid' state in a material $\left(\mathrm{SrCr}_{9} \mathrm{Ga}_{3} \mathrm{O}_{19}\right)$ that consists of corner-shared triangles. Unlike the low-temperature state of an antiferromagnet, the spins in a spinliquid behave like water molecules in the liquid state - when looked at in pairs, there is a preferred mutual arrangement of molecules (or spins), but this arrangement does not extend beyond the nearest neighbour.

Although geometric frustration is being recognized as a new way to classify magnets in naturally occurring minerals such as spinels and pyrochlores, the most common example of the phenomenon is found in ordinary water ice - or 'ice-one'. (There are many different crystal phases of ice, accessible under applied pressure. There is even an ice-nine, although it doesn't possess the properties proposed by Kurt Vonnegut.) Here, despite the well-ordered oxygen lattice, the hydrogen positions are completely disordered, down to the lowest accessible temperatures. This disorder was predicted by Linus Pauling in the 1930s on the basis of missing entropy in specific heat measurements, and has now been observed directly.

'Spin ice' is an analogy between the hydrogen positions in ice and the magnetic moment directions in pyrochlore-based magnets. Like the hydrogen positions in ice-one, there is complete disorder among the magneticmoment directions, despite the fact that they occupy a well-ordered lattice. Unlike ice-one, however, the spin-ice lattice is formed at a temperature that is much higher than that at which the moments interact, which allows the study of ice-like dynamics over a broad range of energies. Thus, although it is of greater practical use, ice-one is the worst system for studying its own dynamics.

Geometric frustration might exist in other non-magnetic materials, such as the ceramic $\mathrm{ZrW}_{2} \mathrm{O}_{8}$. This material exhibits negative thermal expansion: it expands when cooling, while retaining its cubic, symmetrical structure. This is in contrast to most other materials, which not only shrink when cooled, but

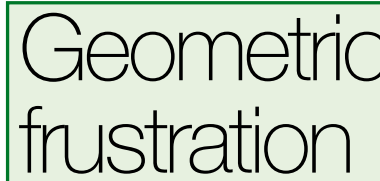

When magnetic moments that prefer anti-alignment reside on a triangular crystal lattice, there is no simple pattern that satisfies all of the bonds.

also adopt a lower-symmetry structure. Such behaviour is accompanied by a brief softening of the lattice, a process absent from $\mathrm{ZrW}_{2} \mathrm{O}_{8}$.

Other structural versions of geometric frustration might exist in materials that possess both a body-centred unit cell and a local atomic tendency to exhibit ferroelectricity, which are mutually incompatible on the grounds of symmetry. Synthesizing such materials is a challenge, but carries potential commercial benefits, in the form of flash memory or smart cards with a variety of uses from toll booths to supermarkets.

When moment interactions fall significantly below the crystallization energy of the structure, as in the above examples, the structure dictates the behaviour at low energies through frustration. However, if the structure itself can respond to the frustration, unexpected patterns emerge. The blue phase of cholesteric liquid crystals is one example. Another is the design of self-assembled structures, such as nanotubules, for drug and gene delivery. Nanotubules are variants of liposomes, lipid electrolyte bilayers that curl up into a spaceenclosing ball. When the polyelectrolytes and oppositely charged lipid membranes have very different charge densities, simple layered structures or balls are not sterically possible, leading to nanotubule formation.

Liposomes have micro-encapsulating and chemical properties that are valuable in textiles, cosmetics and drug delivery, in which specific sites in the body can, in principle, be targeted for administration of small amounts of drugs. Examples are retinoic acid for skin care, an influenza vaccine, and a topical anticancer cream. Fine-tuning the size and shape of nanotubules by understanding the shape-forming mechanisms of geometrical frustration should allow even greater flexibility for designing drugs.

Arthur P. Ramirez is at the Materials Integration Science Laboratory and the Institute for Complex Adaptive Matter, Los Alamos National Laboratory, Los Alamos, New Mexico 87545, USA.

\section{FURTHER READING}

Ramirez, A. P. in Handbook of Magnetic Materials Vol. 13 (ed. Buschow, K. H. J.) 423-520 (Elsevier Science, Amsterdam, 2001).

Harris, M. J. et al. Phys. Rev. Lett. 79, 2554-2557 (1997). Wong, C. C. L. et al. Science 288, 2035-2039 (2000). 